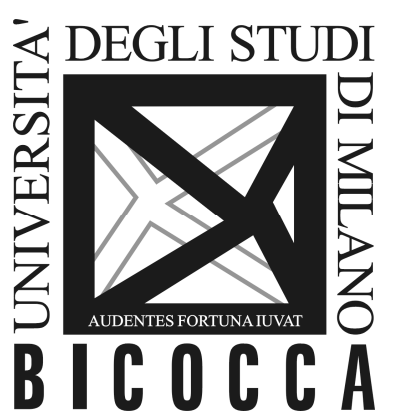

\author{
DEPARTMENT OF ECONOMICS, \\ MANAGEMENT AND STATISTICS \\ UNIVERSITY OF MILAN - BICOCCA
}

DEMS WORKING PAPER SERIES

\title{
An investigation of weak-veto rules in preference aggregation
}

\author{
Stergios Athanasoglou
}

No. 363 - February 2017

Dipartimento di Economia, Metodi Quantitativi e Strategie di Impresa Università degli Studi di Milano - Bicocca

http://dems.unimib.it/ 


\title{
An investigation of weak-veto rules in preference aggregation
}

\author{
Stergios Athanasoglou*
}

January 2017

\begin{abstract}
A new class of preference-aggregation rules is proposed, weak-veto rules. Weak-veto rules are applicable in settings characterized by strong pre-existing views on the desirability of different outcomes, whose recommendations should be modified only in the presence of strenuous opposition. They are characterized by strategy-proofness, strong efficiency, and unanimity-basedness. When non-manipulability requirements are strengthened to $K$-strategy-proofness, the positive results are weakened, except for the case of three alternatives and a sub-class of weak-veto rules based on Kemeny distances. This demonstrates the compatibility of stronger efficiency and non-manipulability properties for the threealternative model.
\end{abstract}

Keywords: social choice, preference aggregation, strategy-proofness, weak-veto rules

JEL classifications: D71, C70

*Department of Economics, University of Milan - Bicocca, athanassoglou@gmail.com. 


\section{Introduction}

Consider the standard Arrovian framework in which agents submit strict linear orderings over a set of alternatives (Arrow [2]). An aggregation rule (or simply a rule) is a function synthesizing the group's preferences into a single such ordering. In contrast to the rich literature on choice rules, where the objective is to pick a single winning alternative (Barbera [5]), the strategic properties of aggregation rules had not been the object of much study until the recent work of Bossert and Sprumont [6].

This was so primarily because comparing orderings of alternatives is not as straightforward as comparing single alternatives. With a few sporadic exceptions (e.g., Bossert and Storcken [7]), researchers had not addressed this issue until Bossert and Sprumont [6] broke important new ground by employing a betweeness relation originally due to Grandmont [8] to compare orderings. Bossert and Sprumont's insight naturally lead to a non-manipulability criterion referred to as strategy-proofness. In their framework, a rule fails strategy-proofness if by manipulating one's preferences it is possible to obtain an outcome that unambiguously dominates the one obtained under truthfulness. Bossert and Sprumont went on to analyze three classes of rules (monotonic majority alteration, status-quo, and Condorcet-Kemeny) on the basis of their efficiency and strategy-proofness properties.

Following up on Bossert and Sprumont's work, Harless [9] studied a generalization of status-quo rules and characterized these rules with novel solidarity axioms. Sato [15] showed that a continuity property known as bounded response combined with strategy-proofness leads to dictatorial rules and impossibility results. Athanasoglou [3] introduced stronger efficiency and nonmanipulability criteria based on Kemeny distances, $K$-strategy-proofness and $K$-efficiency, and demonstrated that (with the exception of Condorcet-Kemeny rules and $K$-efficiency) none of the rules proposed by Bossert and Sprumont satisfy them. Along similar lines, in earlier work Bossert and Storcken [7] 
had established impossibility results regarding the much stronger, coalitional version of $K$-strategy-proofness. ${ }^{1}$

Contribution. The present paper contributes to this growing literature by introducing and axiomatizing a new class of rules, weak-veto rules. These rules use an exogenous ordering over orderings and select the highest-ranked element on this list which, when considering any binary comparison of alternatives, does not result in a violation of unanimous agreement. ${ }^{2}$ The name "weak veto" is due to the fact that a single agent, by promoting or preventing unanimous agreement, may conceivably alter the outcome of the rule (though not necessarily - hence the qualifier "weak").

We show that weak-veto rules are characterized by strategy-proofness, strong efficiency and unanimity-basedness, a property imposing that the rule be robust to changes in preferences that do not alter the structure of unanimous agreement in the electorate. This characterization is tight. As a result of their dependence on patterns of unanimous consent, weak-veto rules are primarily applicable in settings characterized by strong pre-existing views on the desirability of different outcomes, whose recommendations should be modified only if met by strenuous opposition. We argue that this characteristic resonates in

\footnotetext{
${ }^{1}$ Other recent papers (suggestive of the attention this problem is receiving in the literature) focusing on non-strategic aspects of preference aggregation include Baldiga [4], Muto and Sato [13] and Laine, Ozkes and Sanver [11].

${ }^{2}$ In fairness, it should be noted that a sub-class of weak-veto rules (which I call $K$-weakveto rules in Section 4) were mentioned once previously in the literature, in the concluding section of Bossert and Storcken [7], where the authors briefly discuss the tightness of their impossibility results. In an example, the authors claimed that these rules satisfy $K$-coalitional strategy-proofness when the number of alternatives is restricted to three, but no subsequent analysis was done to verify this statement. Though familiar with the Bossert-Storcken paper, I became aware of this fact only after having identified weak-veto rules myself via extensive numerical simulations. These simulations were based on a linear-programming formulation of the existence problem for the case of three alternatives and three/four agents (write-up and Matlab programs available upon request).
} 
several real-life examples of group decision-making, both legal and political.

Weak-veto rules do not in general satisfy the stronger non-manipulability criterion of $K$-strategy-proofness. However, when the number of alternatives is three, we show that a sub-class of weak-veto rules, namely $K$-weak-veto rules, is $K$-strategy-proof. This settles in the affirmative a question posed by Athanasoglou [3] regarding the compatibility of non-dictatoriality, strong efficiency and $K$-strategy-proofness. Since we also show that in the threealternative model strong efficiency is equivalent to $K$-efficiency, a corollary of this analysis is a possibility result regarding non-dictatoriality, $K$-efficiency and $K$-strategy-proofness for the three-alternative model.

The paper is organized as follows. Section 2 introduces the formal model and defines weak-veto rules. Section 3 presents and proves the characterization of weak-veto rules, the paper's main result. It also provides a critical discussion of weak-veto rules, commenting on their practical applicability and axiomatic underpinnings relative to other well-known classes of rules. Section 3 ends with an inquiry into the implications of $K$-strategy-proofness in this setting. Section 4 provides concluding remarks. The Appendix collects all proofs.

\section{Model description}

Let $A=\left\{a_{1}, a_{2}, \ldots, a_{m}\right\}$ denote a finite set of $m \geq 3$ alternatives and $N=$ $\{1,2, \ldots, n\}$ a finite set of $n \geq 2$ agents.

Agents in $N$ submit strict linear orderings ${ }^{3}$ over alternatives in $A$ (i.e., complete, transitive, and antisymmetric binary relations) and the set of such orderings is denoted by $\mathcal{R}$. Given an ordering $R$ and a pair of alternatives $(a, b)$, we use the notation $a R b$ or $(a, b) \in R$ to indicate that $a$ is at least as good as $b$ according to $R$. A preference profile $R_{N}=\left(R_{1}, R_{2}, \ldots, R_{n}\right)$ is an $n$-tuple of orderings, representing the preferences of all agents in $N$ (here, $R_{i}$ denotes the preferences of agent $\left.i \in N\right)$. The set of possible preference

\footnotetext{
${ }^{3}$ From now on simply referred to as "orderings".
} 
profiles is given by $\mathcal{R}^{N}$. An aggregation rule (or simply a rule) is a function $f: \mathcal{R}^{N} \mapsto \mathcal{R}$, assigning to each preference profile an ordering.

Consistent with Grandmont [8], for any $R, R^{\prime}, R^{\prime \prime} \in \mathcal{R}$, we say that $R^{\prime \prime}$ is between $R$ and $R^{\prime}$, and write $R^{\prime \prime} \in\left[R, R^{\prime}\right]$, if and only if $R \cap R^{\prime} \subseteq R^{\prime \prime}$. That is, ordering $R^{\prime \prime}$ agrees with both $R$ and $R^{\prime}$ whenever the latter two agree. Bossert and Sprumont [6] define the prudent extension of an ordering $R \in \mathcal{R}$ as the binary relation $\mathbf{R}$ over orderings given by

$$
R^{\prime \prime} \mathbf{R} R^{\prime} \Leftrightarrow R^{\prime \prime} \in\left[R, R^{\prime}\right], \text { for all } R^{\prime \prime}, R^{\prime} \in \mathcal{R}
$$

Hence, for an agent holding preferences $R, R^{\prime \prime}$ is at least as good as $R^{\prime}$ if and only if $R^{\prime \prime}$ is between $R$ and $R^{\prime}$, and it is strictly better if also $R^{\prime \prime} \neq R^{\prime}$. Since not all pairs of orderings are comparable in this way, the relation $\mathbf{R}$ is a partial order (i.e., a reflexive, transitive, and anti-symmetric binary relation) on $\mathcal{R}$ that is not complete. Indeed, the relation $\mathbf{R}$ ranks one ordering over another if and only if the former unambiguously dominates the latter. As such, it can be thought of as the most conservative (hence the moniker "prudent") relation over orderings that is consistent with an agent having preferences $R$.

Bossert and Sprumont [6] used the above prudent relation to propose the following two concepts of efficiency and non-manipulability.

Efficiency. There do not exist $R_{N} \in \mathcal{R}^{N}$, and $R^{\prime} \in \mathcal{R}$ such that $R^{\prime} \in$ $\left[R_{i}, f\left(R_{N}\right)\right]$ for all $i \in N$ and $R^{\prime} \neq f\left(R_{N}\right)$.

Thus, a rule satisfies efficiency if it produces an ordering such that there exists no other ordering that all agents unambiguously prefer to it. In this sense, it uses the aforementioned prudent extension of orderings to provide an analogue of Pareto efficiency for the economic environment at hand (see Bossert and Sprumont [6] for further discussion of this point).

Before defining strategy-proofness we need to introduce some further notation. Given a profile $R_{N} \in \mathcal{R}^{N}$ and $i \in N$, let $R_{-i}$ denote the orderings of all agents in $N$ except $i$. Subsequently, given $R_{i}^{\prime} \in \mathcal{R}$, define the profile ${ }^{4}$

\footnotetext{
${ }^{4}$ Assume that the obvious adjustments are made if $i=1$ or $i=n$.
} 
$\left(R_{i}^{\prime}, R_{-i}\right) \equiv\left(R_{1}, R_{2}, \ldots, R_{i-1}, R_{i}^{\prime}, R_{i+1}, \ldots, R_{n}\right)$

Strategy-proofness. There do not exist $R_{N} \in \mathcal{R}^{N}, i \in N$ and $R_{i}^{\prime} \in \mathcal{R}$ such that $f\left(R_{i}^{\prime}, R_{-i}\right) \in\left[R_{i}, f\left(R_{N}\right)\right]$ and $f\left(R_{i}^{\prime}, R_{-i}\right) \neq f\left(R_{N}\right)$.

Thus, strategy-proofness ensures that by misreporting one's preferences it is not possible to obtain an ordering that unambiguously dominates that under truthfulness. As such, it imposes a minimal standard of non-manipulability.

An additional property that Bossert and Sprumont discuss is strong efficiency (they refer to it as "local unanimity" - I adopt the arguably bettersuited name used by Harless [9]). Well-known since the seminal work of Arrow [2], strong efficiency applies to preference profiles in which there is unanimous agreement over individual binary comparisons. When such unanimous agreement is present, strong efficiency requires that the rule must also respect its wishes.

Strong efficiency. For all $R_{N} \in \mathcal{R}^{N}$ we have $\bigcap_{i \in N} R_{i} \subseteq f\left(R_{N}\right)$.

Note that strong efficiency implies efficiency (see footnote 11 in Harless [9]). ${ }^{5}$ The reverse direction can be easily seen not to hold. For instance, status-quo rules satisfy efficiency but fail strong efficiency [6, 3].

Given a profile $R_{N} \in \mathcal{R}_{N}$ and a complete, reflexive and anti-symmetric relation $\succeq$ on $A$ (used to break ties if the number of agents is even and there is a 50-50 split), define the majority relation $M_{\succeq}\left(R_{N}\right)$ on $A$ by

$$
\begin{aligned}
M_{\succeq}\left(R_{N}\right) \equiv & \left\{(a, b) \in A \times A:\left|i \in N: a R_{i} b\right|>\left|i \in N: b R_{i} a\right|\right. \\
& \text { or } \left.\left|i \in N: a R_{i} b\right|=\left|i \in N: b R_{i} a\right| \text { and } a \succeq b\right\} .
\end{aligned}
$$

The relation $M_{\succeq}$ is complete and reflexive, and it is anti-symmetric if $n$ is odd. We are now ready to define majority-basedness, a property ascribing primary significance to the majority relation when deriving the social ordering.

\footnotetext{
${ }^{5}$ Note that Bossert and Sprumont [6] contains a typo in this regard, as it claims the two properties are independent.
} 
Majority-basedness. For all $R_{N}, \tilde{R}_{N} \in \mathcal{R}^{N}$

$$
M_{\succeq}\left(R_{N}\right)=M_{\succeq}\left(\tilde{R}_{N}\right) \Rightarrow f\left(R_{N}\right)=f\left(\tilde{R}_{N}\right) .
$$

Majority-based rules thus only retain information regarding the results of majority comparisons between alternatives. There is a plethora of such rules including the all-important class of tournament solutions (Laslier [12], Bossert and Sprumont [6]). Among a multitude of features, they have the important characteristic of coinciding with the majority relation whenever the latter is transitive.

We now introduce a property that is, in a technical sense, similar to majority-basedness. Given a profile $R_{N} \in \mathcal{R}_{N}$, define the unanimity relation $U\left(R_{N}\right)$ on $A$ by

$$
U\left(R_{N}\right) \equiv \bigcap_{i \in N} R_{i}=\left\{(a, b) \in A \times A:\left|i \in N: a R_{i} b\right|=n\right\}
$$

For any profile $R_{N}$, the relation $U\left(R_{N}\right)$ is a partial order (i.e., a reflexive, antisymmetric and transitive binary relation) on $A$. By definition, all strongly efficient orderings are supersets of $U\left(R_{N}\right)$; furthermore, for any pair of alternatives $(a, b) \in A \times A$ inclusion in $U\left(R_{N}\right)$ obviously implies inclusion in $M_{\succeq}\left(R_{N}\right)$, i.e., $a U\left(R_{N}\right) b \Rightarrow a M_{\succeq}\left(R_{N}\right) b$.

Unanimity-basedness. For all $R_{N}, \tilde{R}_{N} \in \mathcal{R}^{N}$,

$$
U\left(R_{N}\right)=U\left(\tilde{R}_{N}\right) \Rightarrow f\left(R_{N}\right)=f\left(\tilde{R}_{N}\right)
$$

Unanimity-basedness stipulates that a rule should be invariant to changes in agent preferences that do not alter the underlying unanimity relation of the electorate. As such, it implies that the rule can be recast as a function from $\mathcal{U}$ to $\mathcal{R}$, where $\mathcal{U}$ is the set of partial orders on $A$. It is straightforward to check that unanimity-basedness implies Bossert and Storcken's [7] extrema independence axiom, and thus also precludes dictatoriality. From a practical standpoint, in Section 3.2 we argue that unanimity-based rules can be found in a number of real-life settings of group decision-making, both legal and political. 
Unanimity-basedness is clearly a strong requirement. It is thus natural to wonder which known rules are able to meet it. Among the strategy-proof rules examined by Bossert and Sprumont [6], status-quo rules are the only ones that are unanimity-based. This can be readily from their definition in Bossert and Sprumont, and also from their reformulation in Eq. (6) of Athanasoglou [3]. ${ }^{6}$

Proposition 1 All status quo rules are unanimity-based.

Proof. See Appendix.

Weak-veto rules. Let us now formally introduce weak-veto rules. Given an ordering $o=R^{1} \succeq R^{2} \succeq \ldots \succeq R^{m !}$ on $\mathcal{R}$, the weak-veto rule $V^{o}$ is defined as the output of the following algorithm:

Input: profile $R_{N}=\left(R_{1}, R_{2}, \ldots, R_{n}\right)$
for $k=1: m !$
if $R^{k} \supseteq U\left(R_{N}\right)$
then $V^{o}\left(R_{N}\right)=R^{k}$. STOP, exit loop.
end
end

Figure 1: Defining weak-veto rule $V^{o}$ for $o=R^{1} \succeq R^{2} \succeq \ldots \succeq R^{m !}$.

Thus, for each profile $R_{N}$ weak-veto rule $V^{o}$ assigns to it the highest-ranked ordering in $o$ satisfying strong efficiency. Since by definition $R_{j} \supseteq U\left(R_{N}\right)$ for all $j \in N$, the above algorithm is well-defined and guaranteed to terminate.

Weak-veto rules can be interpreted in the following way. Suppose ordering $o$ represents a pre-existing consensus view on the relative desirability of different outcomes (in our case, orderings). Moreover, assume the recommendations of ordering $o$ should always be heeded, unless they result in unanimous

\footnotetext{
${ }^{6}$ That monotonic majority alteration rules and Condorcet-Kemeny rules are not unanimity-based is obvious.
} 
opposition on the part of the agents. As we are dealing with orderings, such opposition takes the form of unanimous disagreement regarding individual binary comparisons. Thus, the decision-maker should go down ordering $o$ and pick its highest-ranked element that does not meet such resistance. Section 3.2 offers a more in-depth discussion and interpretation of weak-veto rules and their axiomatic underpinnings, while Section 3.3 compares them to monotonic majority alteration rules and status-quo rules.

\section{The characterization}

\subsection{The main result}

The proof of the main result frequently invokes the following Lemma.

Lemma 1 Suppose rule $f$ satisfies unanimity-basedness and strategy-proofness. Then, for all $R_{N}^{1}, R_{N}^{2} \in \mathcal{R}_{N}$ we have

$$
U\left(R_{N}^{1}\right) \subseteq U\left(R_{N}^{2}\right) \subseteq f\left(R_{N}^{1}\right) \Rightarrow f\left(R_{N}^{1}\right)=f\left(R_{N}^{2}\right) .
$$

Proof. See Appendix.

Lemma 1 is reminiscent of Arrow's choice axiom (Arrow [1]). It implies that if an ordering $R$ is selected from a set of strongly efficient orderings, then $R$ must also be picked when considering any subset of this set for which it does not violate strong efficiency. For example, if $A=\{a, b, c\}$ and rule $f$ picks ordering $R=a b c^{7}$ when there is no unanimous agreement among agents with respect to any pair of alternatives, then it should also pick $R$ when unanimous agreement among agents is confined to everyone ranking $a$ over $b$.

We are now ready to state the paper's main result.

Theorem 1 A rule satisfies strong efficiency, unanimity-basedness, and strategyproofness if and only if it is a weak-veto rule.

\footnotetext{
${ }^{7}$ Where we are using the shorthand notation $R=a_{1} a_{2} a_{3}$ for $R=$ $\left\{\left(a_{1}, a_{1}\right),\left(a_{2}, a_{2}\right),\left(a_{3}, a_{3}\right),\left(a_{1}, a_{2}\right),\left(a_{1}, a_{3}\right),\left(a_{2}, a_{3}\right)\right\}$.
} 
Proof. See Appendix.

Remark 1. The above characterization is tight since (a) Condorcet-Kemeny rules satisfy strong efficiency and strategy-proofness but not unanimity-basedness, (b) status-quo rules satisfy unanimity-basedness and strategy-proofness but not strong efficiency, and (c) we can construct rules satisfying unanimitybasedness and strong efficiency that violate strategy-proofness. The latter can be done by taking a function $f: \mathcal{R}^{N} \mapsto \mathcal{R}$ that respects unanimitybasedness (meaning that it can be recast as $f: \mathcal{U} \mapsto \mathcal{R}$ ) and strong efficiency, but violates Lemma 1 and thus also strategy-proofness. For example, suppose $A=\{a, b, c\}, f$ is unanimity-based and strongly efficient and satisfies $f(\{(a, a),(b, b),(c, c)\})=a b c$ and $f(\{(a, a),(b, b),(c, c),(a, b)\})=c a b .^{8}$ As- $^{-}$ sume, further, that profile $R_{N}$ is such that $U\left(R_{N}\right)=\{(a, a),(b, b),(c, c),(a, b)\}$ so that $f\left(R_{N}\right)=c a b$. Suppose now that agent $i$ has preferences $R_{i}=a b c$. If this agent misreports his preferences submitting $R_{i}^{\prime}=b a c$, we have $U\left(R_{i}^{\prime}, R_{-i}\right)=$ $\{(a, a),(b, b),(c, c)\}$, so that $f\left(R_{i}^{\prime}, R_{-i}\right)=a b c$ and strategy-proofness is violated.

\subsection{Discussion}

It is legitimate to wonder about the relevance, theoretical as well as practical, of the above findings. In addressing this issue one must grapple with two interrelated questions: (i) Are weak-veto rules a desirable way of dealing with the preference aggregation problem? and; (ii) Are their axiomatic underpinnings compelling?

Let us first discuss point (i). It is fair to say that weak-veto rules, with their strong emphasis on unanimity, are not suitable for all settings. For instance, it would be ludicrous to use them as a way of ranking and selecting regular elected representatives, when majority-based rules would be far more

\footnotetext{
${ }^{8}$ Note that $U\left(R_{N}\right)=\{(a, a),(b, b),(c, c)\}$ means that in profile $R_{N}$ there is no unanimous agreement beyond the generic one due to the $R_{i}$ 's reflexivity.
} 
appropriate. Instead, weak-veto rules are appealing in environments characterized by a strong pre-existing understanding of socially desirable outcomes. In such settings, an exogenously-given ordering over outcomes (in our modeling environment, an ordering of orderings) should be consulted closely and its recommendations rejected only if met by extreme resistance. Indeed, the decision-maker should pick the first element on this list that does not, in any way, go against the unanimous wishes of the agents. In this sense, a high bar must be passed before changes are made to pre-existing arrangements, which in turn serves to encourage stability.

An additional motivation for the use of weak-veto rules can be found in their small informational requirements. All that is needed to apply them is information on the unanimity relation on alternatives. In cases like those just mentioned, where there is a strong a priori understanding on the desirability of different outcomes, such simplicity would seem to be in order.

In addressing point (ii), one primarily needs to justify the desirability of unanimity-basedness as an axiom. ${ }^{9}$ While admittedly a very strong property, unanimity-basedness is not without practical relevance. Most famously, criminal law jury trials in the United States and other parts of the world require unanimous agreement to issue a verdict. If such consensus is not reached then a mistrial is declared, with some default outcome typically being implemented. Conversely, in the political sphere, a number of prominent political bodies are known to use unanimity-based methods in their decision-making processes. For example, the five permanent members of the United Nations Security Council (United States, Russia, China, France, United Kingdom) have veto power in the approval of all "substantive" (as opposed to "draft") resolutions. These countries can also use their veto power in the selection of the United Nations' Secretary General. Another pertinent example of unanimity-basedness in political decision-making can be observed in the European Council's deliberative procedures. There, unanimous agreement is needed for decisions to be reached

\footnotetext{
${ }^{9}$ By contrast, strong efficiency and especially strategy-proofness are easier to accept.
} 
in many important areas including EU membership, taxation, foreign policy, common security, justice and home affairs, and many others.

To be sure, the correspondence of these real-life examples to unanimitybased preference aggregation, as discussed in this paper, is not one-to-one. Still, I would argue that it is not too much of a stretch to claim that a relevant connection between theory and practice, worthy of mention, exists.

\subsection{Comparison with other rules}

It is interesting to compare weak-veto rules to two strategy-proof classes of rules discussed by Bossert and Sprumont, namely monotonic majority-alteration rules and status-quo rules.

Monotonic majority alteration rules. Let us begin with monotonic majority alteration rules. These rules are majority-based and satisfy an additional requirement. They take the majority relation and eliminate its possible intransitivities by using a so-called monotonic alteration. For clarity, we provide a brief formal description. Denote by $\mathcal{T}$ the set of tournaments, i.e., the set of complete, reflexive, and anti-symmetric binary relations, on $A$. A monotonic alteration $\phi$ is a function from $\mathcal{T}$ to $\mathcal{R}$ such that for any two distinct tournaments $T_{1}$ and $T_{2}$ we have $T_{2} \in\left[R, T_{1}\right] \Rightarrow \phi\left(T_{1}\right) \notin\left[R, \phi\left(T_{2}\right)\right]$. In words, if a tournament $T_{2}$ is between ordering $R$ and tournament $T_{1}$, then a monotonic alteration cannot reverse this betweenness comparison. Bossert and Sprumont characterized monotonic majority alteration rules with majority-basedness, unanimity (a minimal efficiency requirement imposing that if all agents have the same orderings, then the rule must also pick this ordering), and strategyproofness. Among others, the well-known Slater rule can be classified as a monotonic majority alteration rule.

The relation between the axiomatic foundations of monotonic majority alteration rules and weak-veto rules is worth commenting on. The first thing to note is that the characterization of weak-veto rules significantly strength- 
ens the efficiency requirement from unanimity to strong efficiency. This is not surprising, as Bossert and Sprumont show that some monotonic majority alteration rules fail strong efficiency. Second, in passing from monotonic majority alteration rules to weak-veto rules, majority-basedness is dropped in favor of unanimity-basedness. These two properties are similar in a technical sense, in that they both involve very summary informational requirements, but unanimity-basedness is clearly the more controversial of the two. It is not appropriate in many of the settings that would call for majority-based rules. Rather, as discussed earlier, unanimity-basedness is applicable in decisionmaking contexts where primary emphasis is placed on unanimous consent.

Status-quo rules. Let us now address status-quo rules, arguably the closest relative of weak-veto rules. These rules are similar to weak-veto rules in that they, too, consider exogenous preferences when deriving the social ordering. The key difference lies in the fact that they take into account an exogenous ordering, representing a status-quo outcome to be improved upon, as opposed to an exogenous ordering on orderings. Formally, given $R^{0} \in \mathcal{R}$ and its prudent extension $\mathbf{R}^{0}$, the status-quo rule associated with $R^{0}$ assigns to each profile $R_{N}$ the unique minimal common upper bound of the set of orderings $\left\{R^{0}, R_{1}, R_{2}, \ldots, R_{n}\right\} .{ }^{10}$ That is, it selects the unique ordering $R$ satisfying (i) $R \supseteq R^{0} \cap \bigcup_{i=1}^{n} R_{i}$ and; (ii) $R^{\prime} \supseteq R^{0} \cap \bigcup_{i=1}^{n} R_{i} \Rightarrow R^{\prime} \in\left[R^{0}, R\right]$ for all $R^{\prime} \in \mathcal{R}$ such that $R^{\prime} \neq R$. Athanasoglou [3] showed that this is equivalent to picking the ordering that subsumes $R^{0} \cap \bigcup_{i=1}^{n} R_{i}$ that is simultaneously closest in Kemeny distance ${ }^{11}$ from all the $R_{i}$ 's.

Weak-veto and status-quo rules thus improve on differents sorts of a priori arrangements. The former consider a status-quo ordering of orderings and follow its recommendations to the extent that they do not conflict with strong efficiency -that is, they pick its highest-ranked element to satisfy strong effi-

\footnotetext{
${ }^{10}$ Status-quo rules are reminiscent of the well-known class of median rules in single-peaked social choice (Moulin [14]).

${ }^{11}$ More on Kemeny distances in the following sub-section.
} 
ciency. Conversely, the latter consider a status-quo ordering and seek to improve upon it by taking its minimal common upper bound with the preferences of the agents. This improvement produces an ordering that is simultaneously "closest" to the preferences of all agents, subject to restriction that it subsume the intersection of the status-quo ordering with the union of all agent orderings.

Thus, weak-veto and status-quo rules improve upon exogenously-given recommendations in different ways. How do these differences translate on the axiomatic plane? Clearly, status-quo rules are unanimity-based and they are also efficient. They are not, however, strongly efficient. Instead, they satisfy a property known as population monotonicity which, together with efficiency, characterizes them (Bossert and Sprumont [6], Harless [9]). This property stipulates that the departure of any subset of agents results in a social ordering that all remaining agents find at least as good as the original (according to the prudent relation of their preferences). Furthermore, in a version of the model with a fixed population, Harless [9] showed that status-quo rules are characterized by efficiency and a related solidarity axiom known as adjacent welfare dominance. ${ }^{12}$ An immediate corollary of these results is that weak-veto rules do not, in general, satisfy either population monotonicity or adjacent welfare dominance.

\subsection{Kemeny-based concepts of efficiency and strategy- proofness}

In this section we address the ability of weak-veto rules to satisfy stronger nonmanipulability and efficiency criteria introduced by Athanasoglou [3], namely

\footnotetext{
${ }^{12}$ This is an alternative, more technical, solidarity criterion focusing on the rule's implications when a single agent changes his preferences. It implies that, when this change in preferences is "small" (i.e., the new ordering is adjacent to the original one), then either all other agents find the new ordering at least as good as the original one, or they all find the original one at least as good as the new one.
} 
$K$-strategy-proofness and $K$-efficiency (the prefix $K$ is meant to suggest "Kemeny").

Given two orderings $R, R^{\prime} \in \mathcal{R}$, define the set $D\left(R, R^{\prime}\right)=\left(R \backslash R^{\prime}\right) \cup\left(R^{\prime} \backslash R\right)$, which includes all pairs of alternatives on which $R$ and $R^{\prime}$ disagree. The Kemeny distance [10] between $R$ and $R^{\prime}$, denoted by $\delta\left(R, R^{\prime}\right)$, is given by $\delta\left(R, R^{\prime}\right)=\left|D\left(R, R^{\prime}\right)\right|$. We now formally define $K$-strategy-proofness.

$K$-strategy-proofness. There do not exist $R_{N} \in \mathcal{R}^{N}, i \in N$ and $R_{i}^{\prime} \in \mathcal{R}$ such that $\delta\left(f\left(R_{i}^{\prime}, R_{-i}\right), R_{i}\right)<\delta\left(f\left(R_{N}\right), R_{i}\right)$.

Similarly to the the three classes of strategy-proof rules analyzed by Bossert and Sprumont [6], weak-veto rules do not in general satisfy this strong nonmanipulability requirement (see following Remarks 3 and 4). Nevertheless, when the number of alternatives is limited to three, a certain sub-class of weak-veto rules based on Kemeny distances, $K$-weak-veto rules, does. We proceed to define them.

$K$-weak-veto rules are defined as the sub-class of weak-veto rules $V^{o}$ for which the ordering $o$ satisfies:

(i) There exists $\tilde{R} \in \mathcal{R}$ such that for all $R^{i}, R^{j} \in \mathcal{R}$, the following holds: $\delta\left(\tilde{R}, R^{i}\right)<\delta\left(\tilde{R}, R^{j}\right) \Rightarrow R^{i} \circ R^{j}$.

In words, $o$ assigns highest priority to some $\tilde{R} \in \mathcal{R}$, and orders the remaining orderings in decreasing Kemeny distance to $\tilde{R}$. For each choice of $\tilde{R}$ this operation gives rise to a distinct partial order, which can be subsequently extended to a total order in a number of ways.

Proposition 2 Suppose $m=3$. All $K$-weak-veto rules satisfy $K$-strategyproofness.

Proof. See Appendix.

We now address efficiency. To this end, Athanasoglou [3] used similar Kemeny-based concepts to propose a stronger efficiency property than strong efficiency, $K$-efficiency. 
$K$-efficiency. There do not exist $R_{N} \in \mathcal{R}^{N}$, and $R^{\prime} \in \mathcal{R}$ such that $\delta\left(R^{\prime}, R_{i}\right) \leq$ $\delta\left(f\left(R_{N}\right), R_{i}\right)$ for all $i \in N$ and there exists $j \in N$ such that $\delta\left(R^{\prime}, R_{j}\right)<$ $\delta\left(f\left(R_{N}\right), R_{j}\right)$.

It is easy to see that $K$-efficiency implies strong efficiency (e.g., Proposition 2 in [3]), whereas the opposite direction does not in general hold. Nonetheless, when the number of alternatives is limited to three, it turns out that it does and the two concepts are equivalent.

Proposition 3 When $m=3$, a rule $f$ is strongly efficient if and only if it is K-efficient.

Proof. See Appendix.

A corollary of Theorem 1 and Propositions 2 and 3 is that $K$-weak-veto rules are both $K$-efficient and $K$-strategy-proof for the three-alternative model. Corollary 1 Suppose $m=3$. All $K$-weak-veto rules are $K$-efficient and $K$ strategy-proof.

Remark 2. From previous work we know that when $m=3$, all CondorcetKemeny rules (which are by definition $K$-efficient) satisfy $K$-strategy-proofness on a large profile subdomain, while status-quo rules are $K$-strategy-proof but fail strong efficiency and thus also $K$-efficiency (see Theorems 1 and 2 in [3]). Corollary 1 further extends our understanding of the three-alternative case, demonstrating that there exists a non-dictatorial rule satisfying both $K$ efficiency and $K$-strategy-proofness. This result establishes the compatibility of these three properties for the restricted model, thereby settling a question posed by Athanasoglou [3]. In fact, one may also use the proof of Theorem 1 in Athanasoglou [3] to establish that, when $m=3$, all Condorcet-Kemeny rules whose orderings follow the structure of $o$ in the definition of $K$-weak-veto rules, satisfy $K$-strategy-proofness on the full domain.

Remark 3. Note that Proposition 2 does not necessarily hold for non- $K$ weak-veto rules. For instance, suppose $R_{1}=a b c, R_{2}=a c b, R_{3}=c a b$, and 
$o: b a c \succeq c a b \succeq \ldots$. Since $U\left(R_{N}\right)=\{(a, a),(b, b),(c, c),(a, b)\}$, we will have $f\left(R_{N}\right)=c a b$ yielding $\delta\left(R_{1}, f\left(R_{N}\right)\right)=2$. Now suppose $R_{1}^{\prime}=b a c$. Then, since $U\left(R_{1}^{\prime}, R_{-1}\right)=\{(a, a),(b, b),(c, c)\}$, we will have $f\left(R_{1}^{\prime}, R_{-1}\right)=b a c$, yielding $\delta\left(R_{1}, f\left(R_{1}^{\prime}, R_{-1}\right)\right)=1$ and thus violating $K$-strategy-proofness.

Remark 4. Note, further, that Proposition 2 does not necessarily hold for $m>3 . \quad$ For instance, suppose $R_{1}=c a d b, R_{2}=b a d c, R_{3}=d a b c$, and suppose $f$ is $K$-weak-veto rule such that (i) $d c b a$ is ranked first, and (ii) $a d c b$ is ranked first among orderings having Kemeny distance 3 from $d c b a$. Since $U\left(R_{N}\right)=\{(a, a),(b, b),(c, c),(d, d)\}$, we have $f\left(R_{N}\right)=d c b a$, so that $\delta\left(R_{3}, f\left(R_{N}\right)\right)=3$. Now suppose $R_{3}^{\prime}=$ badc. Then, since $U\left(R_{3}^{\prime}, R_{-3}\right)=$ $\{(a, a),(b, b),(c, c),(d, d),(a, d)\}$, and all orderings $R$ such that $(a, d) \in R$ have Kemeny distance three or more from $d c b a$, we must have $f\left(R_{3}^{\prime}, R_{-3}\right)=a d c b$. This in turn implies that $\delta\left(R_{3}, f\left(R_{3}^{\prime}, R_{-3}\right)=2\right.$, thereby violating $K$-strategyproofness.

Since we can relatively easily find $K$-weak veto rules that fail $K$-strategyproofness, we suspect that no weak-veto rules are $K$-strategy-proof. If this is in fact true then, combined with Theorem 1, it would establish an impossibility result for the $m>3$ case regarding strong efficiency, unanimity-basedness, and $K$-strategy-proofness. It would also suggest that the existence of nontrivial $K$ strategy-proof rules for the general case remains unresolved.

Remark 5. Finally, note that the efficiency component of Corollary 1 is not true for $m>3$ since it is possible to construct $K$-weak-veto rules that violate $K$-efficiency (one may use the example following Proposition 2 in [3] to this effect).

\section{Conclusion}

This paper has been concerned with preference aggregation in the classical Arrovian framework. It proposed a new class of rules, weak-veto rules, and showed that they are the only strategy-proof and strongly efficient rules that 
satisfy an additional invariance property, unanimity-basedness. This characterization is tight. Weak-veto rules are primarily applicable in contexts in which there exists a strong pre-existing view on the desirability of different outcomes that should be revised only if met with strenuous opposition. This feature resonates in several real-life instances of group decision-making, legal as well as political.

When non-manipulability requirements are strengthened to $K$-strategyproofness the positive results are weakened, except for the case of three alternatives and a sub-class of weak-veto rules in which priorities are determined on the basis of Kemeny distances. This possibility result (coupled with the equivalence of strong efficiency and $K$-efficiency for the three-alternative model) demonstrates that when the number of alternatives is limited to three, $K$ strategy-proofness is compatible with a strong efficiency requirement such as $K$-efficiency without needing to resort to dictatorial rules.

The present work suggests several fruitful avenues for future research. An impossibility result regarding $K$-strategy-proofness, strong efficiency, and unanimity-basedness (and perhaps even non-dictatoriality) seems probable. If true, this would mean that the existence of non-trivial $K$-strategy-proof rules remains an open question. Finally, a characterization of strategy-proofness that encompasses all rules known to satisfy it (monotonic majority alteration, status-quo, Condorcet-Kemeny, and weak-veto) would significantly deepen our understanding of non-manipulability in preference aggregation, but seems much harder to come by.

\section{Appendix}

\section{A1: Proofs}

Proposition 1. Consider a profile $R_{N}$ and status-quo ordering $R^{0}$. The status-quo rule associated with $R^{0}$ assigns to each profile $R_{N}$ the unique or- 
dering $R$ satisfying (i) $R \in\left[R^{0}, R_{i}\right]$ for all $i \in N$ and; (ii) $\left\{R^{\prime} \in\left[R^{0}, R_{i}\right]\right.$ for all $i \in N\} \Rightarrow R^{\prime} \in\left[R^{0}, R\right]$ for all $R^{\prime} \in \mathcal{R}$ such that $R^{\prime} \neq R$.

Note that the condition $R \in\left[R^{0}, R_{i}\right]$ for all $i \in N$ is equivalent to requiring $R \supseteq R^{0} \cap \bigcup_{i=1}^{n} R_{i}$. Now, consider two profiles $R_{N}$ and $R_{N}^{\prime}$ such that $U\left(R_{N}\right)=$ $U\left(R_{N}^{\prime}\right)$ and suppose there exists a pair of alternatives $(a, b)$ such that $(a, b) \in$ $\bigcup_{i=1}^{n} R_{i}$ but $(a, b) \notin \bigcup_{i=1}^{n} R_{i}^{\prime}$. This implies that $(b, a) \in U\left(R_{N}^{\prime}\right)$ and $(b, a) \notin$ $U\left(R_{N}\right)$, a contradiction. Thus, we conclude that for all pairs of profiles $R_{N}$ and $R_{N}^{\prime}$ we have $U\left(R_{N}\right)=U\left(R_{N}^{\prime}\right) \Rightarrow \bigcup_{i=1}^{n} R_{i}=\bigcup_{i=1}^{n} R_{i}^{\prime}$. Combining this fact with the definition of status-quo rules mentioned above, we conclude that two profiles having the same unanimity relation will necessarily yield identical outcomes under any status-quo rule.

Lemma 1. Consider any two profiles $R_{N}^{1}, R_{N}^{2}$ satisfying $U\left(R_{N}^{1}\right) \subseteq U\left(R_{N}^{2}\right)$ and $f\left(R_{N}^{1}\right) \supseteq U\left(R_{N}^{2}\right)$. Let $\tilde{R}_{N}^{1}$ be a profile such that there exist two orderings $R_{1}^{1}$ and $R_{2}^{1}$ such that ${ }^{13}$ for all $i \in N$ we have $\tilde{R}_{i}^{1} \in\left\{R_{1}^{1}, R_{2}^{1}\right\}$ and $U\left(\tilde{R}_{N}^{1}\right)=$ $R_{1}^{1} \cap R_{2}^{1}=U\left(R_{N}^{1}\right)$. Similarly, consider a profile $\tilde{R}_{N}^{2}$ such that for all $i \in N$ we have $\tilde{R}_{i}^{2} \in\left\{R_{1}^{2}, R_{2}^{2}\right\}$ and $U\left(\tilde{R}_{N}^{2}\right)=R_{1}^{2} \cap R_{2}^{2}=U\left(R_{N}^{2}\right)$. By Unanimity-basedness we have that $f\left(R_{N}^{1}\right)=f\left(\tilde{R}_{N}^{1}\right)$ and $f\left(R_{N}^{2}\right)=f\left(\tilde{R}_{N}^{2}\right)$.

By the definition of the betweeness relation, we know that $\{R \in \mathcal{R}: R \supseteq$ $\left.R_{1}^{1} \cap R_{2}^{1}\right\}=\left[R_{1}^{1}, R_{2}^{1}\right]$ and $\left\{R \in \mathcal{R}: R \supseteq R_{1}^{2} \cap R_{2}^{2}\right\}=\left[R_{1}^{2}, R_{2}^{2}\right]$. Moreover, since $U\left(\tilde{R}_{N}^{1}\right) \subseteq U\left(\tilde{R}_{N}^{2}\right)$ we have $\left[R_{1}^{1}, R_{2}^{1}\right] \supseteq\left[R_{1}^{2}, R_{2}^{2}\right]$, where we suppose without loss of generality that $R_{1}^{1} \cap R_{1}^{2} \supseteq R_{1}^{1} \cap R_{2}^{2}$ so that $R_{1}^{2}$ has more elements in common with $R_{1}^{1}$ than $R_{2}^{2}$ does. Now, consider a profile $\hat{R}_{N}$ where for all $i \in N$ we have $\hat{R}_{i} \in\left[R_{1}^{1}, R_{2}^{2}\right]$ and $U\left(\hat{R}_{N}\right)=R_{1}^{1} \cap R_{2}^{2}$, implying that we also have $U\left(\hat{R}_{N}\right) \supseteq U\left(\tilde{R}_{N}^{1}\right) .{ }^{14}$ We argue that $f\left(\hat{R}_{N}\right)=f\left(\tilde{R}_{N}^{1}\right)$. Suppose not, so that

\footnotetext{
${ }^{13}$ This is a slight abuse of notation since $R_{1}^{1}$ and $R_{2}^{1}$ do not necessarily belong in profile $R_{N}^{1}$.

${ }^{14}$ If the reader finds this notation hard to follow, an illustrative example would be $\left[R_{1}^{1}, R_{2}^{1}\right]=[c d a b, b a c d], \quad\left[R_{1}^{2}, R_{2}^{2}\right]=[c b d a, c b a d]$, and $\left[R_{1}^{1}, R_{2}^{2}\right]=[c d a b, c b a d], \quad$ so that $U\left(\tilde{R}_{N}^{1}\right)=\{(a, a),(b, b),(c, c),(d, d),(c, d)\}$, $U\left(\tilde{R}_{N}^{2}\right)=\{(a, a),(b, b),(c, c),(d, d),(c, d),(c, b),(c, a),(b, d),(b, a)\}$, and $U\left(\hat{R}_{N}\right)=$
} 
$f\left(\hat{R}_{N}\right) \neq f\left(\tilde{R}_{N}^{1}\right)$. Distinguish now between two cases according to whether in profile $\hat{R}_{N}$ (a) there exists an agent $i \in N$ having preferences $\hat{R}_{i}=f\left(\tilde{R}_{N}^{1}\right)$, or (b) not. If (a) holds, suppose this agent $i$ misreports his preferences to $R_{i}^{\prime}=R_{2}^{1}$ so that $U\left(R_{i}^{\prime}, \hat{R}_{-i}\right)=U\left(\tilde{R}_{N}^{1}\right)$. By unanimity-basedness, $f\left(R_{i}^{\prime}, \hat{R}_{-i}\right)=f\left(\tilde{R}_{N}^{1}\right)$ violating strategy-proofness. Thus, we must have $f\left(\hat{R}_{N}\right)=f\left(\tilde{R}_{N}^{1}\right)$. If case (b) is true, so that in profile $\hat{R}_{N}$ no agent $i$ has preferences $\hat{R}_{i}=f\left(\tilde{R}_{N}^{1}\right)$, then define a profile $\bar{R}_{N}^{1}$ satisfying $U\left(\bar{R}_{N}^{1}\right)=U\left(\hat{R}_{N}\right)$ in which such an agent exists and use unanimity-basedness to deduce that $f\left(\hat{R}_{N}\right)=f\left(\bar{R}_{N}^{1}\right)$. Then consider profile $\bar{R}_{N}^{1}$ and use the same argument as before to derive a violation of strategy-proofness for the agent holding preferences $f\left(\tilde{R}_{N}^{1}\right)$ to conclude that $f\left(\bar{R}_{N}^{1}\right)=f\left(\tilde{R}_{N}^{1}\right)$. This in turn implies $f\left(\hat{R}_{N}\right)=f\left(\tilde{R}_{N}^{1}\right)$.

We focus now on profiles $\hat{R}_{N}$ and $\tilde{R}_{N}^{2}$. Again, distinguish between two cases according to whether in profile $\tilde{R}_{N}^{2}$ (a) there exists $i \in N$ such that $\tilde{R}_{i}^{2}=f\left(\hat{R}_{N}\right)=f\left(\tilde{R}^{1}\right)$, or (b) not. If case (a) holds, suppose this agent $i$ misreports his preferences to $R_{i}^{\prime}=R_{1}^{1}$ so that $U\left(R_{i}^{\prime}, \tilde{R}_{-i}^{2}\right)=U\left(\hat{R}_{N}\right)$. As a result, $f\left(R_{i}^{\prime}, \tilde{R}_{-i}^{2}\right)=f\left(\hat{R}_{N}\right)$ violating strategy-proofness. Thus, we must have $f\left(\hat{R}_{N}\right)=f\left(\tilde{R}_{N}^{2}\right)$. On the other hand, if case (b) holds and such an agent does not exist, introduce a profile $\bar{R}_{N}^{2}$ in which it does and use Unanimity-basedness to repeat the same argument as before to conclude that $f\left(\hat{R}_{N}\right)=f\left(\bar{R}_{N}^{2}\right)=$ $f\left(\tilde{R}_{N}^{2}\right)$. Together with previously established $f\left(\hat{R}_{N}\right)=f\left(\tilde{R}_{N}^{1}\right)$ this implies $f\left(\tilde{R}_{N}^{1}\right)=f\left(\tilde{R}_{N}^{2}\right)$, which in turn yields $f\left(R_{N}^{1}\right)=f\left(R_{N}^{2}\right)$.

Theorem 1. We begin with the easier " $\Rightarrow$ " direction. That weak-veto rules satisfy strong efficiency and unanimity-basedness is obvious by construction. Let us address strategy-proofness. Suppose $f$ is a weak-veto rule with order $R^{1} \succeq R^{2} \succeq \ldots \succeq R^{m !}$. Let $R_{N} \in \mathcal{R}^{N}$ and suppose $f\left(R_{N}\right)=R^{k}$. By strong efficiency this means that $U\left(R_{N}\right) \subseteq R^{k}$. Suppose that $f$ fails strategy-proofness at $R_{N}$. This implies that there exist $i \in N$ and $R_{i}^{\prime}$ such that $f\left(R_{i}^{\prime}, R_{-i}\right) \in$ $\overline{\{(a, a),(b, b),(c, c),(d, d),(c, d),(c, a)},(c, b)\}$. 
$\left[R_{i}, R^{k}\right]$ and $f\left(R_{i}^{\prime}, R_{-i}\right) \neq R^{k}$. Suppose $f\left(R_{i}^{\prime}, R_{-i}\right)=R^{l}$, where $l \neq k$. Thus, we have $U\left(R_{-i}\right) \cap R_{i}^{\prime} \subseteq R^{l}$. Let us distinguish between two cases:

(i) $l<k$. Hence, $U\left(R_{-i}\right) \cap R_{i}^{\prime} \subseteq R^{l}$ and $U\left(R_{N}\right) \nsubseteq R^{l}$. Since $U\left(R_{N}\right) \subseteq R^{k}$, there must exist a pair of alternatives $(x, y) \in R_{i}$ such that $(x, y) \in R^{k}$ and $(x, y) \notin R^{l}$. This contradicts $R^{l} \in\left[R_{i}, R^{k}\right]$.

(ii) $l>k$. Hence, $U\left(R_{-i}\right) \cap R_{i}^{\prime} \subseteq R^{l}$ and $U\left(R_{-i}\right) \cap R_{i}^{\prime} \nsubseteq R^{k}$. Since $U\left(R_{N}\right) \subseteq$ $R^{k}$, there must exist a pair of alternatives $(x, y) \in R_{i}^{\prime}$ such that $(x, y) \notin$ $R_{i},(x, y) \notin R^{k}$ and $(x, y) \in R^{l}$. Thus, $(y, x) \in R_{i},(y, x) \in R^{k}$, and $(y, x) \notin R^{l}$. This contradicts $R^{l} \in\left[R_{i}, R^{k}\right]$.

We now turn to the " $\Leftarrow$ " direction. Recall that $\mathcal{U}$ denote the set of partial orders of $A$. By unanimity-basedness, it is possible to recast the rule $f$ as a function from $\mathcal{U}$ to $\mathcal{R}$. So, we have $f: \mathcal{U} \mapsto \mathcal{R}$, where $f(U)=f\left(R_{N}\right)$ for all $R_{N} \in \mathcal{R}^{N}$ such that $U\left(R_{N}\right)=U$. Consequently, we re-write Lemma 1 to suppress its dependence on profiles: For any two $U^{1}, U^{2} \in \mathcal{U}$, we have

$$
U^{1} \subseteq U^{2} \subseteq f\left(U^{1}\right) \Rightarrow f\left(U^{1}\right)=f\left(U^{2}\right)
$$

Note that rule $f$ induces a binary relation $\succeq^{f}$ on $\mathcal{R}$ defined as follows

$$
R^{1} \succeq^{f} R^{2} \Leftrightarrow \exists U \in \mathcal{U} \text { such that } R^{1}, R^{2} \supseteq U \text { and } f(U)=R^{1} .
$$

It must be stressed that $\succeq^{f}$ is not necessarily complete. This means that it is possible to have a pair of orderings such that $R^{1} \nsucceq^{f} R^{2}$ and $R^{2} \nsucceq^{f} R^{1}$.

We use $\succeq^{f}$ to define the binary relation $\succeq^{f^{*}}$ on $\mathcal{R}$ in the following way:

1. $R^{1} \succeq^{f} R^{2} \Rightarrow R^{1} \succeq f^{*} R^{2}$, for all $R^{1}, R^{2} \in \mathcal{R}$, and

2. $\left\{R^{1} \succeq^{f} R^{2}\right.$ and $\left.R^{2} \succeq^{f} R^{3}\right\} \Rightarrow R^{1} \succeq^{f^{*}} R^{3}$, for all distinct $R^{1}, R^{2}, R^{3} \in$ $\mathcal{R}$.

We will show that $\succeq^{f^{*}}$ is a partial order (i.e., a reflexive, transitive, and antisymmetric binary relation) on $\mathcal{R}$. 
That $\succeq^{f^{*}}$ is reflexive is obvious (for any ordering $R \in \mathcal{R}$ just apply $U=R$ to the right-hand side of Eq. (6) and impose strong efficiency on $f$ ). Let us show it is also anti-symmetric. Suppose there exist $U \in \mathcal{U}$ and $R^{1}, R^{2} \in \mathcal{R}$ such that $f(U)=R^{1}$ and $R^{1}, R^{2} \supseteq U$. This implies that $R^{1} \succeq^{f^{*}} R^{2}$. Suppose, further than there exists $\tilde{U} \in \mathcal{U}$ such that $f(\tilde{U})=R^{2}$ and $R^{1}, R^{2} \supseteq \tilde{U}$. This implies $R^{2} \succeq^{f^{*}} R^{1}$. Consider the partial order $R^{1} \cap R^{2}$. Since $R^{1} \supseteq U$ and $R^{2} \supseteq U$, we have $U \subseteq R^{1} \cap R^{2}$. Similarly, we have $\tilde{U} \subseteq R^{1} \cap R^{2}$. Applying Eq. (5) to $U$ and $R^{1} \cap R^{2}$, we have $R^{1}=f(U)=f\left(R^{1} \cap R^{2}\right)$. Doing the same for $\tilde{U}$ and $R^{1} \cap R^{2}$ implies $R^{2}=f(\tilde{U})=f\left(R^{1} \cap R^{2}\right)$. Hence, $R^{1}=R^{2}$.

We now show that $\succeq^{f^{*}}$ satisfies transitivity. We do this by showing that for any three distinct orderings $R^{1}, R^{2}$, and $R^{3}$ we have $\left\{R^{1} \succeq^{f} R^{2}\right.$ and $R^{2} \succeq^{f}$ $\left.R^{3}\right\} \Rightarrow R^{3} \nsucceq^{f} R^{1}$. Suppose otherwise. Thus, there exist $U^{1}, U^{2}, U^{3} \in \mathcal{U}$ such that:

(a) $f\left(U^{1}\right)=R^{1}$, and $R^{1}, R^{2} \supseteq U^{1}$.

(b) $f\left(U^{2}\right)=R^{2}$, and $R^{2}, R^{3} \supseteq U^{2}$.

(c) $f\left(U^{3}\right)=R^{3}$, and $R^{3}, R^{1} \supseteq U^{3}$.

The anti-symmetry of relation $\succeq^{f^{*}}$ immediately implies that $R^{3} \nsupseteq U^{1}, R^{1} \nsupseteq$ $U^{2}$, and $R^{2} \nsupseteq U^{3}$. Moreover, by similar reasoning as before, we must have $f\left(R^{1} \cap R^{2}\right)=R^{1}, f\left(R^{2} \cap R^{3}\right)=R^{2}$, and $f\left(R^{3} \cap R^{1}\right)=R^{3}$. To avoid immediate contradictions via Eq. (5), we must have $R^{3} \notin\left[R^{1}, R^{2}\right], R^{1} \notin\left[R^{2}, R^{3}\right]$ and $R^{2} \notin\left[R^{1}, R^{3}\right]$.

Consider the partial order $R^{1} \cap R^{2} \cap R^{3}$. We distinguish between two cases:

(i) $f\left(R^{1} \cap R^{2} \cap R^{3}\right)=R^{k} \in\left\{R^{1}, R^{2}, R^{3}\right\}$. Here, applying Eq. (5) to partial orders $R^{1} \cap R^{2} \cap R^{3}$ and $R^{k-1} \cap R^{k}$ (defining $R^{k-1} \equiv R^{3}$ when $k=1$ ), we obtain $f\left(R^{1} \cap R^{2} \cap R^{3}\right)=R^{k}=f\left(R^{k-1} \cap R^{k}\right)=R^{k-1}$, a contradiction.

(ii) $f\left(R^{1} \cap R^{2} \cap R^{3}\right)=R^{l} \notin\left\{R^{1}, R^{2}, R^{3}\right\}$. The definition of the betweeness relation implies the following:

$$
\left\{R \in \mathcal{R}: R \supseteq R^{1} \cap R^{2} \cap R^{3}\right\}=\left\{R \in \mathcal{R}: R \in\left[R^{1}, R^{2}\right] \cup\left[R^{2}, R^{3}\right] \cup\left[R^{3}, R^{1}\right]\right\}
$$


Thus by strong efficiency $R^{l}=f\left(R^{1} \cap R^{2} \cap R^{3}\right) \in\left[R^{k-1}, R^{k}\right]$ for some $k=1,2,3$ (again, defining $R^{k-1} \equiv R^{3}$ when $k=1$ ), so that $f\left(R^{1} \cap R^{2} \cap\right.$ $\left.R^{3}\right) \supseteq R^{k-1} \cap R^{k}$. Applying Eq. (5) to $R^{1} \cap R^{2} \cap R^{3}$ and $R^{k-1} \cap R^{k}$ we obtain $f\left(R^{1} \cap R^{2} \cap R^{3}\right)=R^{l}=f\left(R^{k-1} \cap R^{k}\right)=R^{k-1}$, a contradiction.

Thus, we have showed that for any three distinct orderings $R^{1}, R^{2}$, and $R^{3}$ we have $\left\{R^{1} \succeq^{f} R^{2}\right.$ and $\left.R^{2} \succeq^{f} R^{3}\right\} \Rightarrow R^{3} \succeq^{f} R^{1}$. As a result, the statement $\left\{R^{1} \succeq^{f^{*}} R^{2}\right.$ and $\left.R^{2} \succeq^{f^{*}} R^{3}\right\} \Rightarrow R^{1} \succeq^{f^{*}} R^{3}$ results in no contradiction, and we conclude that $\succeq^{f^{*}}$ is transitive.

Hence, $\succeq^{f^{*}}$ is a partial order on $\mathcal{R}$. By the order-extension principle it can be extended to a total order (i.e., an ordering), call it $o^{f^{*}}$. Now, let $g$ be a weak-veto rule having ordering $o^{f^{*}}$. We will argue that $f=g$. Take any profile $R_{N}$ and let $f\left(R_{N}\right)=f\left(U\left(R_{N}\right)\right)=R^{l}$. By Eq. (6) we know that ordering $o^{f^{*}}$ is such that $R^{l}$ is preferred to, and thus ranked first among, all orderings in the set $\left\{R \in \mathcal{R}: R \supseteq U\left(R_{N}\right)\right\}$. As a result, we must also have $g\left(R_{N}\right)=R^{l}=f\left(R_{N}\right)$.

Proposition 2. Let $A=\{a, b, c\}$. Suppose $g$ is a $K$-weak-veto rule with an ordering $o$. Let $R_{N} \in \mathcal{R}^{N}$. Suppose, without loss of generality, that $R_{i}=a b c$ and that there exists $R_{i}^{\prime} \in \mathcal{R}$ such that $\delta\left(R_{i}, g\left(R_{N}\right)\right)>\delta\left(R_{i}, g\left(R_{i}^{\prime}, R_{-i}\right)\right)$. We distinguish between 4 cases:

(i) $\delta\left(R_{i}, g\left(R_{N}\right)\right)=0$. Since $0 \leq \delta\left(R_{i}, R\right)$ for all $R \in \mathcal{R}$, we immediately reach a contradiction.

(ii) $\delta\left(R_{i}, g\left(R_{N}\right)\right)=1$. Thus, we must have $\delta\left(R_{i}, g\left(R_{i}^{\prime}, R_{-i}\right)\right)=0$. Hence, $R_{i}=g\left(R_{i}^{\prime}, R_{-i}\right)$. This implies that rule $g$ is not strategy-proof which contradicts Theorem 1 .

(iii) $\delta\left(R_{i}, g\left(R_{N}\right)\right)=3$. Thus, we must have $\delta\left(R_{i}, g\left(R_{i}^{\prime}, R_{-i}\right)\right)<3$. Then, it must be the case that $g\left(R_{N}\right)=c b a$ and $g\left(R_{i}^{\prime}, R_{-i}\right) \neq c b a$. This again contradicts the strategy-proofness of $g$. 
(iv) $\delta\left(R_{i}, g\left(R_{N}\right)\right)=2$. This is the only nontrivial case and we address it in what follows.

Then $\delta\left(R_{i}, g\left(R_{i}^{\prime}, R_{-i}\right)\right)<2$. If $\delta\left(R_{i}, g\left(R_{i}^{\prime}, R_{-i}\right)\right)=0$, then $g\left(R_{i}^{\prime}, R_{-i}\right)=R_{i}$ contradicting strategy-proofness. Suppose instead that $\delta\left(R_{i}, g\left(R_{i}^{\prime}, R_{-i}\right)\right)=1$. Then either $g\left(R_{N}\right)=c a b$ or $g\left(R_{N}\right)=b c a$. Suppose without loss of generality that $g\left(R_{N}\right)=c a b$ (the proof for case $g\left(R_{N}\right)=b c a$ is similar). Then, since the rule is strategy-proof, $g\left(R_{i}^{\prime}, R_{-i}\right)=b a c$. We now argue how this leads to a contradiction.

Distinguish between two cases:

(i) bac $\supseteq U\left(R_{N}\right)$. Then $U\left(R_{N}\right)=\{(a, a),(b, b),(c, c)\}$, so $c a b$ must be ranked first in $o$. Thus, since $b a c$ is the ordering that is farthest away from $c a b$ and as such has lowest priority in $o$, unless $R_{j}=b a c$ for all $j \neq i$ and also $R_{i}^{\prime}=b a c$, we must have $g\left(R_{i}^{\prime}, R_{-i}\right) \neq b a c$. But if that were true, then by strong efficiency $g\left(R_{N}\right) \in\{a b c, b a c\}$, contradicting $g\left(R_{N}\right)=c a b$.

(ii) $b a c \nsupseteq U\left(R_{N}\right)$. Then, it must be that $R_{j} \in\{a b c, a c b, c a b\}$ for all $j \neq i$. This means that there must exist at least one $j \neq i$ such that $R_{j}=c a b$. Thus, to make sure that both $\left.b a c, c a b \supseteq U\left(R_{i}^{\prime}, R_{-i}\right)\right)$ it must be that $\left.U\left(R_{i}^{\prime}, R_{-i}\right)\right)=\{(a, a),(b, b),(c, c)\}$. In which case bac must be ranked first by $o$. But if that were the case, then $g\left(R_{N}\right) \neq c a b$ because $a b c$ is closer in Kemeny distance to bac than $c a b$ is. Again, we reach a contradiction.

Proposition 3. Proposition 2 in Athanasoglou [3] establishes that $K$-efficiency implies strong efficiency. We now focus on the other direction. Let $R_{N} \in \mathcal{R}_{N}$ and suppose rule $f$ satisfies strong efficiency. We distinguish between 4 cases.

(a) $\left|U\left(R_{N}\right)\right|=6$. This implies that for all $i \in N$ we have $R_{i}=R$ for some $R$. By strong efficiency, we must have $f\left(R_{N}\right)=R$. Since this choice coincides with the preferences of all agents, $f$ satisfies $K$-efficiency. 
(b) $\left|U\left(R_{N}\right)\right|=5$. This implies that for all $i \in N$ we have $R_{i} \in\left\{R^{1}, R^{2}\right\}$ for some $R^{1}, R^{2}$ such that $\delta\left(R^{1}, R^{2}\right)=1$, and there exist at least two agents having different orderings. By strong efficiency, we must have $f\left(R_{N}\right) \in\left\{R^{1}, R^{2}\right\}$. Since this choice coincides with the preferences of at least one agent, $f$ satisfies $K$-efficiency.

(c) $\left|U\left(R_{N}\right)\right|=4$. This implies that for all $i \in N$ we have $R_{i} \in\left\{R^{1}, R^{2}, R^{3}\right\}$ for some $R^{1}, R^{2}, R^{3}$ such that $\delta\left(R^{1}, R^{2}\right)=1, \delta\left(R^{2}, R^{3}\right)=1$, and $\delta\left(R^{1}, R^{3}\right)=$ 2 , and there exist at least two agents having orderings $R^{1}$ and $R^{3}$, respectively. By strong efficiency, we must have $f\left(R_{N}\right) \in\left\{R^{1}, R^{2}, R^{3}\right\}$. If $f\left(R_{N}\right) \in\left\{R^{1}, R^{3}\right\}$, or if $f\left(R_{N}\right)=R^{2}$ and there exists an agent with ordering $R^{2}$, then $f\left(R_{N}\right)$ coincides with the preferences of some agent. Hence, the rule satisfies $K$-efficiency. Otherwise, if $f\left(R_{N}\right)=R^{2}$ and no agent has ordering $R^{2}$, we have $\delta\left(f\left(R_{N}\right), R_{i}\right)=1$ for all $i \in N$. Clearly, it is not possible to find another ordering $\tilde{R} \neq f\left(R_{N}\right)$ such that $\delta\left(R_{i}, \tilde{R}\right) \leq \delta\left(R_{i}, f\left(R_{N}\right)\right)$ for all $i \in N$ with strict inequality for at least one $i$. Thus, $f$ satisfies $K$-efficiency.

(d) $\left|U\left(R_{N}\right)\right|=3$. This implies that either (i) there exist $i, j \in N$ such that $\delta\left(R_{i}, R_{j}\right)=3$, or (ii) there is no such pair but there exist $i, j, k \in N$ such that $\delta\left(R_{i}, R_{j}\right)=\delta\left(R_{j}, R_{k}\right)=\delta\left(R_{k}, R_{i}\right)=2$ and $R_{i}, R_{j}, R_{k}$ are all distinct. In both cases, we have $U\left(R_{N}\right)=\{(a, a),(b, b),(c, c)\}$ so that strong efficiency imposes no restrictions on the value of $f\left(R_{N}\right)$. However, this is also true of $K$-efficiency: for any choice of $f\left(R_{N}\right)$, it is not possible to find an ordering $\tilde{R} \neq f\left(R_{N}\right)$ such that $\delta\left(R_{i}, \tilde{R}\right) \leq \delta\left(R_{i}, f\left(R_{N}\right)\right)$ for all $i \in N$ with strict inequality for at least one $i$. Thus, $K$-efficiency is generically satisfied.

We conclude that $f$ is $K$-efficient. 


\section{References}

[1] Arrow, K. J. (1959). Rational choice functions and orderings. Economica, 26, 121-127.

[2] Arrow, K. J. (1963). Social choice and individual values. 2nd edition. John Wiley. New York.

[3] Athanasoglou, S. (2016). Strategyproof and efficient preference aggregation with Kemeny-based criteria. Games and Economic Behavior, 95, 156-167.

[4] Baldiga, K. (2016). Representative democracy and the implementation of majoritypreferred alternatives. Social Choice and Welfare, 46, 477-494.

[5] Barbera, S. (2011). Strategyproof social choice. In: Arrow, K.J., Sen, A., Sen K. (Eds), Handbook of Social Choice and Welfare, vol 2. Elsevier, Amsterdam, pp. 731-831. Chapter 25 .

[6] Bossert, W., and Sprumont, Y. (2014). Strategy-proof preference aggregation: possibilities and characterizations. Games and Economic Behavior, 85, 109-126.

[7] Bossert, W., and Storcken, T. (1992). Strategy-proofness of social welfare functions: the use of the Kemeny distance between preference orderings. Social Choice and Welfare, $9(4), 345-360$.

[8] Grandmont, J. M. (1978). Intermediate preferences and the majority rule. Econometrica, 317-330.

[9] Harless, P. (2016). Solidarity in preference aggregation: Improving on a status quo. Games and Economic Behavior, 95, 73-87.

[10] Kemeny, J.G. (1959). Mathematics without Numbers. Daedalus, 88, 575-591.

[11] Laine, J., Ozkes, A. I., and Sanver, R. (2016). Hyper-stable social welfare functions. Social Choice and Welfare, 46, 157-182.

[12] Laslier, J. F. (1997). Tournament solutions and majority voting (No. 7). Springer Verlag.

[13] Muto, N., and Sato, S. (2016). Bounded response of aggregated preferences. Journal of Mathematical Economics, 65, 1-15.

[14] Moulin, H. (1980). On strategy-proofness and single peakedness. Public Choice, 35(4), 437-455.

[15] Sato, S. (2015). Bounded response and the equivalence of nonmanipulability and independence of irrelevant alternatives. Social Choice and Welfare, 44(1), 133-149. 